\title{
Investigation of a Carbapenemase-producing Acinetobacter baumannii outbreak using whole genome sequencing versus a standard epidemiologic investigation
}

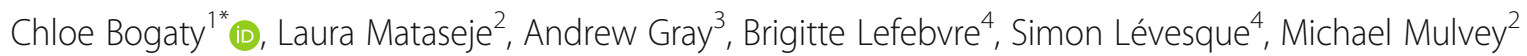
and Yves Longtin ${ }^{5^{*}}$

\begin{abstract}
Background: The standard epidemiologic investigation of outbreaks typically relies on spatiotemporal data and pulsed-field gel electrophoresis (PFGE), but whole genome sequencing (WGS) is becoming increasingly used. This investigation aimed to characterize a carbapenemase-producing Acinetobacter baumannii (CPAb) nosocomial outbreak using WGS compared to a standard outbreak investigation.
\end{abstract}

Methods: The CPAb outbreak occurred in a single center between 2012 and 2014. The standard investigation used spatiotemporal data and PFGE to generate a chain of transmission. A separate WGS investigation generated a chain of transmission based solely on WGS and date of sampling and was blinded to all other spatiotemporal data and PFGE. Core single nucleotide variant (SNV) phylogenetic analysis was performed on WGS data generated using the Illumina MiSeq platform. The chains of transmission were compared quantitatively and qualitatively to assess the concordance between both methods.

Results: 28 colonized and infected cases were included. Of the 27 transmission events identified using the standard investigation, 12 (44\%) were identical to the transmission events using WGS. WGS identified several transmission events that had not been detected by traditional method, and numerous transmission events that had occurred on different hospital wards than suspected by standard methods. The average number (standard deviation [SD]) of SNVs per transmission events was $1.63(\mathrm{SD}, 1.31)$ by traditional method and $0.63(\mathrm{SD}, 0.79)$ by WGS $(p=0.001)$ All isolates harbored the rare carbapenemase bla OXA-237.

Conclusions: The traditional and WGS investigations had moderate concordance. When used alongside epidemiologic data and clinical information, WGS could help improve the mapping of transmission events.

Keywords: Outbreak investigations, Whole-genome sequencing, Acinetobacter baumannii, Carbapenemase-producing organisms, Single nucleotide variant analysis

\footnotetext{
* Correspondence: chloe.bogaty@gmail.com; yves.longtin@mcgill.ca

Presented in part at the 27th European Congress of Clinical Microbiology and

Infectious Diseases (ECCMID), Vienna, Austria. April 2017. Abstract number 3737.

'University of Manitoba, Winnipeg, MB, Canada

${ }^{5}$ McGill University, Montreal, QC, Canada

Full list of author information is available at the end of the article
}

(c) The Author(s). 2018 Open Access This article is distributed under the terms of the Creative Commons Attribution 4.0 International License (http://creativecommons.org/licenses/by/4.0/), which permits unrestricted use, distribution, and reproduction in any medium, provided you give appropriate credit to the original author(s) and the source, provide a link to the Creative Commons license, and indicate if changes were made. The Creative Commons Public Domain Dedication waiver (http://creativecommons.org/publicdomain/zero/1.0/) applies to the data made available in this article, unless otherwise stated. 


\section{Background}

Carbapenemase-producing Acinetobacter baumannii (CPAb) is of global concern [1]. Canada has had infrequent cases [2], but has not been spared from the occasional outbreak [3]. The most recent reported outbreak occurred from 2012 to 2014 in a primary and tertiary institution in Montreal (Canada) [4].

Control of CPAb outbreaks requires meticulous investigation. A standard epidemiologic investigation is a multi-step process including the creation of a chain of transmission [4]. Phenotypic characteristics such as biotypes, serotypes, and antimicrobial susceptibilities are used to infer pathogen inter-relatedness and is combined with spatiotemporal information to determine transmission events [5]. This information is sometimes combined with molecular typing methods (commonly pulsed-field gel electrophoresis (PFGE)). PFGE is usually considered the gold standard for its ability to detect differences across the entire genome $[5,6]$.

Whole genome sequencing (WGS) is increasingly being recognized as a powerful tool for epidemiologic studies. Its ability to distinguish strains that differ by single nucleotides provides high levels of resolution [7, 8]. WGS has been employed in outbreak investigations, both retrospectively to identify environmental sources [9] and additional cases [10], but also in real-time to confirm the existence of an outbreak [11] and track the emergence and spread of mutational resistance mechanisms [12]. However, only a few studies have compared standard epidemiologic outbreak investigations with investigations performed by WGS. Through the subsequent use of WGS, Kanamori et al observed that two epidemiologically unlinked outbreaks were in fact genetically connected [13]. In another study, WGS allowed for the identification of unexpected transmission routes not initially suggested by the epidemiologic data [14].

The aim of this study was to contrast the validity and accuracy of a standard epidemiologic outbreak investigation with one achieved using WGS. We retrospectively characterized the CPAb nosocomial outbreak that occurred in an academic institution in Montreal (Canada) using only WGS analysis and compared the predicted chain of transmission with an existing standard epidemiologic investigation.

\section{Methods}

\section{Setting and definitions}

A CPAb outbreak occurred in a 637-bed primary and tertiary care hospital between March 2012 and January 2014 [4]. A confirmed case was defined as any patient found to be colonized or infected during this period by the outbreak strain, as confirmed by pulsed-field gel electrophoresis interpretative criteria [15].

\section{Standard epidemiologic investigation}

Screening was performed using Acinetobacter-selective chromogenic agar (CHROMagar Acinetobacter, CHROMagar Microbiology, Paris, France). While initially carried out in patients who had shared hospital rooms with known cases, screening eventually evolved to include all patients sharing a ward with a case. Organism identification was conducted using matrix-assisted laser desorption/ionization time-of-flight mass spectrometry (MALDI-TOF MS, bioMérieux, Marcy l'Étoile, France) and antimicrobial susceptibility testing with disc diffusion and E-test following the Clinical and Laboratory Standards Institute breakpoints (2014 M100S). Strain clonality was confirmed by PFGE. Bacterial genomes were digested with ApaI restriction enzyme and samples were run on a CHEF-DR III system (Bio-Rad, Mississauga, ON, Canada). Banding patterns were analyzed using Tenover's criteria [15]. Detection of resistance genes was performed at the National Microbiology Laboratory in Winnipeg (Canada) [16]. Determining the chain of transmission followed a previously described approach, using a combination of spatiotemporal patient information, date of strain isolation, antibiogram results, and PFGE interpretations [4].

\section{DNA extraction and whole genome sequencing}

Total cellular deoxyribonucleic acid (DNA) was prepared using Epicentre MasterPure ${ }^{\mathrm{Tm}}$ Complete kits (Mandel Scientific, Guelph, ON). Libraries were created with TruSeq Nano DNA HT sample preparation kits (Illumina, San Diego, CA). Paired-end, 301 bp indexed reads were generated on an Illumina MiSeq ${ }^{\mathrm{Tm}}$ platform (Illumina, San Diego, CA). De novo assembly of Illumina reads were done using Spades v3.5. Assembled sequence data was analysed using the batch uploader mode at the Centre for Genomic Epidemiology website (https://cge.cbs.dtu.dk/ services/) and data was produced from the ResFinder, PlasmidFinder, VirulenceFinder, and MLST tools.

\section{Single nucleotide variant (SNV) analysis and creation of WGS-based chain of transmission}

SNV analysis was conducted using a previously published pipeline [17]. The following parameters were added: map/base quality 30 , alternate fraction 0.75 , minimum read coverage of identification of variants $10, \mathrm{SNV}$ density filtering of 2 SNVs within a 20-base pair window. The reference strain used was an assembled genome of one of the internal isolates to the outbreak (patient 29). Maximum likelihood phylogenetic trees were built using PhyML v3.1 using parameters "-quiet $-\mathrm{b}-4-\mathrm{m}$ GTR -s BEST" [18]. Images were rendered in FigTree (v1.4.1) (http://tree.bio.ed.ac.uk/software/figtree).

A WGS-based chain of transmission was generated using only the sequencing data and the date of sampling to determine directionality, with complete blinding of 
the epidemiologic data. A cut-off of $\leq 2 \mathrm{SNV}$ was selected to indicate a putative transmission event between patients. Also, when $>1$ potential source patient was plausible for a given transmission event, transmission events with a lower number of SNVs were considered more likely (e.g. $0 \mathrm{SNV}$ more likely than $2 \mathrm{SNVs}$ ).

\section{Comparison of the outbreak investigation methods}

To compare the chains of transmission generated by standard epidemiologic investigation vs. WGS, four different strategies were used. Firstly, both chains of transmission were compared qualitatively. For each transmission event, epidemiologic data was revised to assess where patients had likely acquired the outbreak strain. Secondly, WGS was used to verify the plausibility of each transmission event determined by the standard investigation. Transmission events that remained unchanged were deemed to be supported by WGS, while those that differed were considered unsupported. Thirdly, quantitative SNV differences were calculated by comparing the mean number of SNVs per transmission event between the two investigation methods using the Student's T-test. Statistical significance was defined as a $p$-value of $<0.05$. Finally, the WGS-generated data was compared to that obtained by PFGE for each isolate.

\section{Results}

\section{Outbreak description}

Twenty-eight cases were identified during the outbreak. The index case occurred in the intensive care unit in March 2012. Twenty-seven additional cases were detected up to 2014. Five cases developed CPAb bacteremia, and all died within $72 \mathrm{~h}$ of the first positive blood culture. These represented the only cases of infection, while the remaining 22 cases were only colonized. All isolates were resistant to penicillins, carbapenems, quinolones, and aminoglycosides. Four pulsovars were identified by PFGE. Further details on patient characteristics and the course of the outbreak have been reported previously [4].

\section{Genomic analysis}

By in silico sequence typing, all 28 isolates were found to be of sequence type (ST) 208. Resistant genes identified by WGS were consistent for all isolates. Carbapenem resistance was attributable to the presence of the acquired class $\mathrm{D}$ carbapenemase bla $a_{\mathrm{OXA}-237}$ as well as the intrinsic bla $a_{\text {OXA-66. }}$ Aminoglycoside resistance was attributable to the presence of $\operatorname{aadA1}, \operatorname{aph}\left(3^{\prime}\right)-\operatorname{Ia}, \operatorname{arm} A, \operatorname{str} A$ and $\operatorname{str} B$. Quinolone resistance was due to mutations in both GyrA (Ser-83-Leu) and ParC (Ser-80-Leu) [19, 20].

The total percentage of valid and included positions used for SNV analysis represented $96.8 \%$ of the total core genome. A total of $20 \mathrm{SNVs}$ were used to generate phylogeny as they were identified across all outbreak strains. These $20 \mathrm{SNV}$ loci were extracted and aligned for comparison (Table 1).

\section{Comparison of epidemiologic investigations}

Transmission events using a standard outbreak investigation [4] versus WGS-generated chain of transmission are found in Fig. 1. Of the 27 transmission events determined using a traditional investigation strategy, 12 (44.4\%) were identical to transmission events predicted by WGS, while 15 (55.5\%) were discordant (Fig. 1b).

In the standard investigation, patient 2 was predicted to be the central source for the two principal transmission

Table 1 Single nucleotide variant (SNV) differences between outbreak strains

\begin{tabular}{|c|c|c|c|c|}
\hline \multirow[b]{2}{*}{ Year } & \multirow[b]{2}{*}{ Identifier } & \multirow[b]{2}{*}{$\begin{array}{l}\text { PFGE } \\
\text { Pulsovar }\end{array}$} & \multicolumn{2}{|l|}{ SNV Analysis } \\
\hline & & & Pseudo-alignment & $\begin{array}{l}\text { Difference in } \\
\text { SNVs from } \\
\text { Index Case }\end{array}$ \\
\hline \multirow[t]{4}{*}{2012} & 1 & A5-C & CGCAAGCTTCTGGTTTAACC & Index case \\
\hline & 2 & A & CACAAGACTCTGGTTCAACC & 4 \\
\hline & 3 & A5 & CGCAAGACTCTGGTITAACC & 2 \\
\hline & 4 & A4 & CGCCAGCTTCTGATTTAACC & 2 \\
\hline \multirow[t]{23}{*}{2013} & 5 & A5 & CGCAAGACTCTGGTTTAACC & 2 \\
\hline & 6 & A5 & CGCAAGACTCCGGTITAACC & 3 \\
\hline & 7 & A5 & CGCAAGACTCCGGTITAACC & 3 \\
\hline & 8 & A5 & CGCAAGACTCCGGTGTAACC & 4 \\
\hline & 9 & A5 & CGCAAGACTCCGGTTTAGCC & 4 \\
\hline & 10 & A5 & CGCAAGACTCCGGTTTAACC & 3 \\
\hline & 11 & A5 & CGCAAGACCCTAGTTTAACC & 4 \\
\hline & 12 & A5 & CGCAAGACTCCGGTTTAACC & 3 \\
\hline & 13 & A4 & CGCATAACTCCGGTITAACC & 5 \\
\hline & 14 & A6 & CGCAAAACTCCGGTITAACC & 4 \\
\hline & 15 & A4 & CGCATAACTCCGGTITAACC & 5 \\
\hline & 16 & A6 & CGCATAACTCCGGTITAACC & 5 \\
\hline & 17 & A5 & CGCAAGACTCTGGTTAAACC & 2 \\
\hline & 18 & A5 & TGTAAGACTCTGGTTTAACC & 4 \\
\hline & 19 & A5-a & CGCAAGACTCTGGTTTAACC & 2 \\
\hline & 20 & A5-a & CGCAAGACTCTGGTITAACC & 2 \\
\hline & 21 & A5-a & CGCAAGACTCTGGTTTAACC & 2 \\
\hline & 22 & A5-a & CGCAAGACTCTGGTITAACC & 2 \\
\hline & 23 & A5-a & CGCAAGACTCTGGTTAACC & 2 \\
\hline & 24 & A5 & TGTAAGACTCTGGTTTAACC & 4 \\
\hline & 25 & A5 & CGCAAGACTCTGGTTAATC & 3 \\
\hline & 26 & A5 & TGTAAGACTCTGGTTTAACC & 4 \\
\hline & 27 & A5 & TGTAAGACTCTGGTTTAACC & 4 \\
\hline 2014 & 28 & A6 & CGCAAGACTCTGGATTCACC & 4 \\
\hline
\end{tabular}

Bolded letters represent a SNV difference compared to the index case Abbreviations: SNV, single nucleotide variants; PFGE, pulsed-field gel electrophoresis; $Q$, quarter 


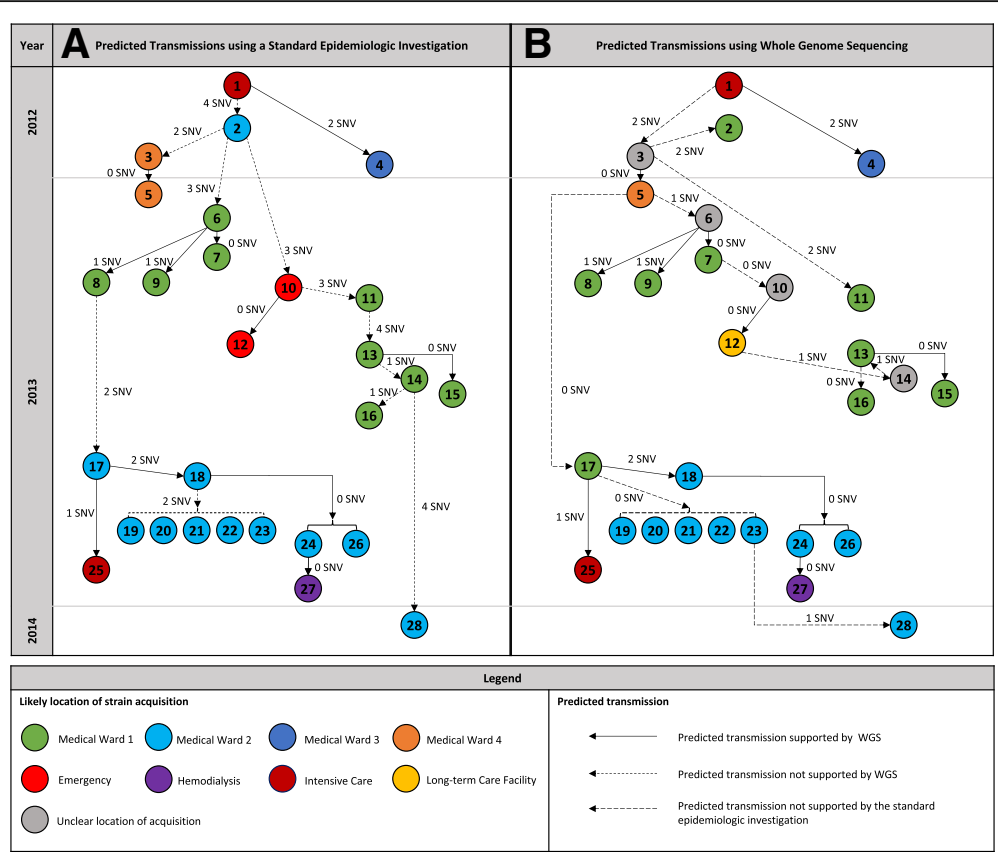

Fig. 1 Transmission of a carbapenemase-producing Acinetobacter baumannii during a hospital outbreak in Montreal, Canada (axis not to scale). (a) Standard epidemiologic investigation, where predicted transmissions were determined using spatiotemporal patient information, date of strain isolation, antibiogram results, and pulsed-field gel electrophoresis interpretation. (b) Whole genome sequencing investigation, where predicted transmissions were determined using single nucleotide variant analysis and the date of strain isolation. Colours indicate the case's location at the time of the predicted exposure. Dashed arrows indicate transmissions that were not supported by the other investigation method. SNV, single nucleotide variant

arms (Fig. 1a). The cluster of transmission that occurred on Medical Ward (MW) 1 in the first quarter of 2013 (patients 6-9) was believed to have been introduced by patient 2. WGS analysis, however, did not support this conclusion and suggested that the strain from patient 6 was more closely related to patient 5 than patient 2 (1 SNV vs $3 \mathrm{SNV}$, respectively). Similarly, the cluster on MW 2 in 2013 (patient 17-24, and 26) was initially believed to have originated from patient 8 , whereas WGS suggested that the strain in this cluster was identical to the strain of patients 3 and $5(0 \mathrm{SNV})$ and different from patient 8 (2 SNVs). Using WGS, patient 3 was deemed central to the outbreak, contributing both to clusters on MW 1 in early-to-mid 2013 and the cluster on MW 2 in late 2013 (Fig. 1b). On the other hand, the WGS-generated chain of transmission also incorrectly identified some transmission events. For example, the source of patient 17 was attributed to patient 5 by WGS and date of sampling, but considering that the strains of patients 3,5 and 17 were identical, the source of patient 17 may have been patient 3 or an unknown intermediate case. In fact, spatiotemporal analysis indicated that patients 3 and 17 were both temporally located on MW 1 prior to patient 17 being transferred to MW 2 where the outbreak strain rapidly disseminated. Other notable discrepancies between both investigation methods included transmissions to patients $6,10,11$, 13 and 28 (Fig. 1).

In the standard investigation, 26 of the 28 cases were found to have shared a ward with a potential source case. [4] In the WGS-generated chain of transmission, 23 of the 27 transmission events could be explained by patients sharing a ward with a known case, though acquisition of the outbreak strain occasionally occurred on a different ward than previously suspected (Fig. 1b).

Two discordant events differed only in terms of directionality. Using standard epidemiologic tools, patient 2 was thought to have transmitted the outbreak strain to patient 3, and patient 13 to 14 . However, WGS suggested that the opposite had actually occurred, postulating that patients 3 and 14 had either acquired the outbreak strain at an earlier date than originally thought or that there were undetected carriers.

SNV differences between transmission events in the standard epidemiologic investigation ranged from 0 to 4 SNVs, while the range was from 0 to $2 \mathrm{SNVs}$ in the WGS-generated chain of transmission. The largest SNV difference between the index case and a subsequent case was $19 \mathrm{SNVs}$ in the standard investigation (index case and patient 28; Fig. 1a) compared to 5 SNVs in the WGS investigation (index case and patients 13, 15 and 16; Fig. 1b). The average number of SNVs 
(standard deviation) per transmission event was 1.63 $(1.31)$ and $0.63 \quad(0.79)$ for the standard vs. WGS-generated investigations, respectively $(p=0.001$ by Student's T-test).

\section{Comparison of conventional typing by PFGE and genomic analysis}

Four pulsovars were identified by PFGE. By WGS, patients 3, 5, 17, and 19 to 23 had no SNV differences. However, PFGE established different band patterns, initially grouping the isolates into pulsovar A5 or A5-a. Examination of these sequences identified three small DNA segments unique to the earlier isolates, but none which explained the band variation seen on PFGE. It is suspected that a genetic event (insertion, deletion or recombination) occurred at or near the restriction site for ApaI causing different band numbers or shifting of band sizes. SNV analysis may not have detected this change as it may have occurred at a site of multiple events outside of our SNV parameters (i.e. $96.8 \%$ of core genome covered by WGS) or was a large event not captured by the SNV workflow. Similarly, PFGE grouped isolates 13 and 15 into pulsovar $\mathrm{A} 4$, and isolate 16 to pulsovar A6, but there were no SNV differences between these 3 strains by WGS.

\section{Discussion}

Outbreak investigations typically rely on spatiotemporal data and PFGE, but WGS is also becoming increasingly used. Our study compared the chains of transmission of a CPAb outbreak using a standard epidemiologic investigation and a WGS-based technique. Using qualitative and quantitative comparative strategies, our investigation identified many similarities between both investigations, but also major differences. Nearly $50 \%$ of the predicted transmissions were identical between both methods, and upon review of the epidemiologic data it was found that several transmissions had occurred on different hospital wards than previously assumed.

The extent of these differences was unexpected. Some discrepancies may be explained by the fact that transmission events in standard epidemiologic investigations are typically deduced based on commonality between a source and a case and are prone to missing transmission events between patients with little spatiotemporal commonality $[5,21]$. The location where transmission occurred could not be identified for four patients from the WGS-generated chain after review of the epidemiologic data; these transmission events were at risk of being missed using traditional tools. Additionally, PFGE detected different band patterns among genetically related isolates by WGS, likely influencing some of the dissimilar epidemiologic predictions. Overall, the chain of transmission determined by WGS might be perceived as more accurate due to the close genetic relatedness of the strains. However, some WGS-generated transmission events may be incorrect: upon reviewing spatiotemporal data, the origin of patient 17 is probably patient 3 instead of patient 5 .

Both chains did retain an important degree of concordance, which is noteworthy considering that the WGS-generated chain of transmission was fully blinded to any spatiotemporal information and to the PFGE typing results. It provides a glimpse of the potential for automated outbreak investigations. Some transmission events determined by traditional method were shown to be implausible by WGS given the high number of SNVs between the source and the recipient. However, it can be argued that some discrepancies are of minor importance, such as linking cases $19-23$ to either patient 17 or 18 . Knowing these differences would have changed little in terms of outbreak confinement measures.

Still, some observations suggested that WGS may have had practical value had it been available. Firstly, the WGS investigation suggests that infection control strategies around patient 3 had failed, leading to the cluster of cases on MW 2. Patients 3 and 17 had been hospitalized on the same ward prior to 17 being diagnosed with the outbreak strain, but this association was not captured in the original investigation. Had it been, there could have been efforts to understand why control measures were breached. Secondly, the SNV analysis suggested missed opportunities for earlier case findings. Real-time WGS-assisted investigation could have suggested the presence of additional unidentified cases. Finally, it was unclear where four cases acquired the outbreak strain. Had the connection between source and case been made in real-time, it might have assisted in generating hypotheses regarding transmission.

WGS is already used for public health surveillance in various jurisdictions [22-25], and has helped perform accurate contact tracing in health-care settings [11, 26, 27]. Our findings further support the potential role of WGS in hospital outbreak investigations, particularly as the technology becomes more affordable and accessible [6, 8, 28, 29].

Two additional observations are noteworthy. Firstly, our investigation demonstrated surprising discrepancies in the phylogenetic assessment of clonality between PFGE and WGS. While PFGE occasionally lacks discriminatory power with closely related bacterial strains $[6,30,31]$, it seemed unusual for it to assign different pulsovars to strains that were found to have no SNV differences. However, A. baumannii can undergo significant horizontal gene transfer and genetic recombination, even over short time periods [32]. Other studies have observed low discriminatory powers of PFGE when applied to A. baumannii [27, 33]. With A. baumannii, PFGE may be too discriminatory between isolates and distort the interpretation of phylogenetic differences, 
essentially leading to "false-negative" results where WGS data may provide indistinguishable differences [7]. SNV analyses may be superior to PFGE for A. baumannii typing as it ignores changes due to horizontal gene transfers and genomic recombination.

Secondly, this study represents the third reported identification of the acquired class D carbapenemase $b l a_{\mathrm{OXA}-237}$, and its first identification in Canada. The $b l a_{\text {OXA-235-like }}$ subclass, which includes $b l a_{\text {OxA-237, }}$, was first recognised from $A$. baumannii isolates in the United States and Mexico in 2013 [34] and was implicated in a multi-center outbreak in Oregon between 2012 and 2014 [35]. This resistance gene was located on a plasmid found in the most prominent $A$. baumannii clonal group, representing a potential for extensive dissemination [36].

Our study has some limitations. Due to its retrospective nature, it remains unknown whether performing WGS in real-time would have altered the outbreak progression. Despite all the discrepancies observed, the standard epidemiologic investigation was ultimately successful in controlling the outbreak. Also, review of the available epidemiologic data could not account for the method of CPAb acquisition in four cases. Subsequent epidemiologic investigation was not conducted to link these cases with a source. A. baumannii can contaminate the hospital environment, such that transmission through fomites or health care workers is possible [3, 27]. Neither investigation can account for the presence of additional unidentified colonized patients [37]. Finally, the lack of a standardized definition for "bacterial clones" in molecular epidemiology for $A$. baumannii also complicates WGS investigations [7, 33, 38-40]. Bacteria naturally undergo genomic diversification, and SNVs can accumulate at a rate of $2-10$ /year in most bacterial genomes [7, 27]. Still, our data demonstrates minimal genetic diversification between isolates, with no more than 5 SNV differences from the index case and some with 0 SNV changes over nearly a year.

\section{Conclusion}

This study suggests that an investigation based on WGS and date of sampling was able to create a transmission sequence that was in many aspects similar to a traditional outbreak investigation and that detected transmission events that had not been suspected using traditional tools. WGS may help improve the understanding of transmission during outbreaks when used alongside epidemiologic data and clinical information.

\section{Abbreviations}

CPAb: Carbapenemase-producing Acinetobacter baumanii; DNA: Deoxyribonucleic acid; MALDI-TOF MS: Matrix-assisted laser desorption/ionization time-of-flight mass spectrometry; MW: Medical ward; PFGE: Pulse-field gel electrophoresis; SNV: Single nucleotide variant; WGS: Whole genome sequencing

\section{Acknowledgements}

We thank all the members of the infection control unit and clinical microbiology laboratory for their dedication in controlling this outbreak.

\section{Funding}

This work was supported by institutional grants and by the Fonds de Recherche du Québec en Santé.

\section{Availability of data and materials}

The datasets used/or analyzed during the current study are available from the corresponding author on reasonable request.

\section{Authors' contributions}

$\mathrm{CB}$ and $\mathrm{YL}$ reviewed and analyzed the molecular data in comparison to the epidemiologic data, and equally contributed to the writing of the manuscript. APG collected and interpreted the epidemiologic data to generate the original outbreak tree. LM and MRM performed the genomic analysis of the outbreak strains using whole-genome sequencing and were major contributors in writing of the manuscript. BL and SL identified the different pulsovars using pulse-field gel electrophoresis and contributed to the epidemiologic investigation of this outbreak. All authors read and approved the final manuscript.

Ethics approval and consent to participate

Jewish General Hospital Research Review Office - \# CODIM-MBM-CR18-05.

Consent for publication

Not applicable.

\section{Competing interests}

$Y L$ reported receiving research funding from Merck and Becton Dickinson. The authors declare that they have no competing interests.

\section{Publisher's Note}

Springer Nature remains neutral with regard to jurisdictional claims in published maps and institutional affiliations.

\section{Author details}

${ }^{1}$ University of Manitoba, Winnipeg, MB, Canada. ${ }^{2}$ National Microbiology Laboratory, Winnipeg, MB, Canada. ${ }^{3}$ Northern Health, Prince George, BC, Canada. ${ }^{4}$ Laboratoire de santé publique du Québec, Sainte-Anne-de-Bellevue, QC, Canada. ${ }^{5}$ McGill University, Montreal, QC, Canada.

Received: 28 August 2018 Accepted: 12 November 2018

Published online: 21 November 2018

\section{References}

1. Peleg AY, Seifert H, Paterson DL. Acinetobacter baumannii: emergence of a successful pathogen. Clin Microbiol Rev. 2008;21:538-82.

2. Mataseje LF, Bryce E, Roscoe D, Boyd DA, Embree J, Gravel D, Katz K, Kibsey $\mathrm{P}$, Kuhn M, Mounchili A, et al. Carbapenem-resistant gram-negative bacilli in Canada 2009-10: results from the Canadian nosocomial infection surveillance program (CNISP). J Antimicrob Chemother. 2012;67:1359-67.

3. Simor AE, Lee M, Vearncombe M, Jones-Paul L, Barry C, Gomez M, Fish JS, Cartotto RC, Palmer R, Louie M. An outbreak due to multiresistant Acinetobacter baumannii in a burn unit: risk factors for acquisition and management. Infect Control Hosp Epidemiol. 2002;23:261-7.

4. Gray AP, Allard R, Pare R, Tannenbaum T, Lefebvre B, Levesque S, Mulvey M, Maalouf L, Perna S, Longtin Y. Management of a hospital outbreak of extensively drug-resistant Acinetobacter baumannii using a multimodal intervention including daily chlorhexidine baths. J Hosp Infect. 2016;93:29-34.

5. Singh A, Goering RV, Simjee S, Foley SL, Zervos MJ. Application of molecular techniques to the study of hospital infection. Clin Microbiol Rev. 2006;19: 512-30

6. Koser CU, Ellington MJ, Cartwright EJ, Gillespie SH, Brown NM, Farrington M, Holden MT, Dougan G, Bentley SD, Parkhill J, Peacock SJ. Routine use of microbial whole genome sequencing in diagnostic and public health microbiology. PLoS Pathog. 2012;8:e1002824.

7. Salipante SJ, SenGupta DJ, Cummings LA, Land TA, Hoogestraat DR, Cookson BT. Application of whole-genome sequencing for bacterial strain typing in molecular epidemiology. J Clin Microbiol. 2015;53:1072-9. 
8. Robinson ER, Walker TM, Pallen MJ. Genomics and outbreak investigation: from sequence to consequence. Genome Med. 2013;5:36.

9. Reuter S, Harrison TG, Koser CU, Ellington MJ, Smith GP, Parkhill J, Peacock SJ, Bentley SD, Torok ME. A pilot study of rapid whole-genome sequencing for the investigation of a legionella outbreak. BMJ Open. 2013;3:e002175.

10. Butcher $H$, Elson $R$, Chattaway MA, Featherstone $C A$, Willis $C$, Jorgensen $F$, Dallman TJ, Jenkins C, MC LJ, Beck CR, Harrison S. Whole genome sequencing improved case ascertainment in an outbreak of Shiga toxinproducing Escherichia coli 0157 associated with raw drinking milk. Epidemiol Infect. 2016;144:2812-23.

11. Eyre DW, Golubchik T, Gordon NC, Bowden R, Piazza P, Batty EM, Ip CL,

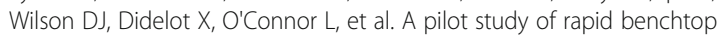
sequencing of Staphylococcus aureus and Clostridium difficile for outbreak detection and surveillance. BMJ Open. 2012;2:e001124.

12. Otter JA, Doumith M, Davies F, Mookerjee S, Dyakova E, Gilchrist M, Brannigan ET, Bamford K, Galletly T, Donaldson H, et al. Emergence and clonal spread of colistin resistance due to multiple mutational mechanisms in carbapenemase-producing Klebsiella pneumoniae in London. Sci Rep. 2017:7:12711.

13. Kanamori H, Parobek CM, Juliano JJ, van Duin D, Cairns BA, Weber DJ, Rutala WA. A prolonged outbreak of KPC-3-producing Enterobacter cloacae and Klebsiella pneumoniae driven by multiple mechanisms of resistance transmission at a large academic burn center. Antimicrob Agents Chemother. 2017;61:e01516.

14. Snitkin ES, Zelazny AM, Thomas PJ, Stock F, Group NCSP, Henderson DK Palmore TN, Segre JA. Tracking a hospital outbreak of carbapenem-resistant Klebsiella pneumoniae with whole-genome sequencing. Sci Transl Med. 2012:4:148ra116.

15. Tenover FC, Arbeit RD, Goering RV, Mickelsen PA, Murray BE, Persing DH, Swaminathan B. Interpreting chromosomal DNA restriction patterns produced by pulsed-field gel electrophoresis: criteria for bacterial strain typing. J Clin Microbiol. 1995;33:2233-9.

16. Woodford N, Ellington MJ, Coelho JM, Turton JF, Ward ME, Brown S, Amyes SG, Livermore DM. Multiplex PCR for genes encoding prevalent OXA carbapenemases in Acinetobacter spp. Int J Antimicrob Agents. 2006;27:351-3.

17. Petkau A, Mabon P, Sieffert C, Knox NC, Cabral J, Iskander M, Iskander M, Weedmark K, Zaheer R, Katz LS, et al. SNVPhyl: a single nucleotide variant phylogenomics pipeline for microbial genomic epidemiology. Microb Genom. 2017;3:e000116.

18. Guindon S, Dufayard JF, Lefort V, Anisimova M, Hordijk W, Gascuel O. New algorithms and methods to estimate maximum-likelihood phylogenies: assessing the performance of PhyML 3.0. Syst Biol. 2010;59:307-21.

19. Vila J, Ruiz J, Goni P, Marcos A, Jimenez de Anta T. Mutation in the gyrA gene of quinolone-resistant clinical isolates of Acinetobacter baumannii. Antimicrob Agents Chemother. 1995:39:1201-3.

20. Vila J, Ruiz J, Goni P, Jimenez de Anta T. Quinolone-resistance mutations in the topoisomerase IV parC gene of Acinetobacter baumannii. J Antimicrob Chemother. 1997;39:757-62.

21. Lesson 6: Investigating an outbreak. In: Principles of Epidemiology in Public Health Practice, Third edition: an introduction to applied epidemiology and biostatistics; 2012.

22. Inns T, Ashton PM, Herrera-Leon S, Lighthill J, Foulkes S, Jombart T, Rehman $Y$, Fox A, Dallman T, E DEP, et al. Prospective use of whole genome sequencing (WGS) detected a multi-country outbreak of Salmonella Enteritidis. Epidemiol Infect. 2016;145:1-10.

23. Joensen KG, Scheutz F, Lund O, Hasman H, Kaas RS, Nielsen EM, Aarestrup FM. Real-time whole-genome sequencing for routine typing, surveillance, and outbreak detection of verotoxigenic Escherichia coli. J Clin Microbiol. 2014;52:1501-10

24. Agren EC, Wahlstrom H, Vesterlund-Carlson C, Lahti E, Melin L, Soderlund R. Comparison of whole genome sequencing typing results and epidemiological contact information from outbreaks of Salmonella Dublin in Swedish cattle herds. Infect Ecol Epidemiol. 2016;6:31782.

25. Mellmann A, Harmsen D, Cummings CA, Zentz EB, Leopold SR, Rico A, Prior K, Szczepanowski R, Ji Y, Zhang W, et al. Prospective genomic characterization of the German enterohemorrhagic Escherichia coli O104:H4 outbreak by rapid next generation sequencing technology. PLoS One. 2011;6:e22751.

26. Koser CU, Holden MT, Ellington MJ, Cartwright EJ, Brown NM, Ogilvy-Stuart AL, Hsu LY, Chewapreecha C, Croucher NJ, Harris SR, et al. Rapid wholegenome sequencing for investigation of a neonatal MRSA outbreak. N Engl J Med. 2012;366:2267-75.
27. Kanamori H, Parobek CM, Weber DJ, van Duin D, Rutala WA, Cairns BA, Juliano JJ. Next-generation sequencing and comparative analysis of sequential outbreaks caused by multidrug-resistant Acinetobacter baumannii at a large academic burn center. Antimicrob Agents Chemother. 2015;60:1249-57.

28. Didelot X, Bowden R, Wilson DJ, Peto TEA, Crook DW. Transforming clinical microbiology with bacterial genome sequencing. Nat Rev Genet. 2012;13: $601-12$.

29. Kwong JC, McCallum N, Sintchenko V, Howden BP. Whole genome sequencing in clinical and public health microbiology. Pathology. 2015;47:199-210.

30. Leekitcharoenphon P, Nielsen EM, Kaas RS, Lund O, Aarestrup FM. Evaluation of whole genome sequencing for outbreak detection of Salmonella enterica. PLoS One. 2014;9:e87991.

31. Foley SL, Zhao S, Walker RD. Comparison of molecular typing methods for the differentiation of Salmonella foodborne pathogens. Foodborne Pathog Dis. 2007:4:253-76.

32. Valenzuela JK, Thomas $L$, Partridge $S R$, van der Reijden T, Dijkshoorn $L$, Iredell J. Horizontal gene transfer in a polyclonal outbreak of carbapenemresistant Acinetobacter baumannii. J Clin Microbiol. 2007;45:453-60.

33. Fitzpatrick MA, Ozer EA, Hauser AR. Utility of whole-genome sequencing in characterizing Acinetobacter epidemiology and analyzing hospital outbreaks. J Clin Microbiol. 2016;54:593-612.

34. Higgins PG, Perez-Llarena FJ, Zander E, Fernandez A, Bou G, Seifert H. OXA-235, a novel class $D$ beta-lactamase involved in resistance to carbapenems in Acinetobacter baumannii. Antimicrob Agents Chemother. 2013;57:2121-6.

35. Buser GL, Cassidy PM, Cunningham MC, Rudin S, Hujer AM, Vega R, Furuno JP, Marshall SH, Higgins PG, Jacobs MR, et al. Failure to communicate: transmission of extensively drug-resistant Bla OXA-237-containing Acinetobacter baumannii-multiple facilities in Oregon, 2012-2014. Infect Control Hosp Epidemiol. 2017;38:1-7.

36. Hujer AM, Higgins PG, Rudin SD, Buser GL, Marshall SH, Xanthopoulou K, Seifert H, Rojas LJ, Domitrovic TN, Cassidy PM, et al. Nosocomial outbreak of extensively drug-resistant Acinetobacter baumannii isolates containing blaOXA-237 carried on a plasmid. Antimicrob Agents Chemother. 2017;61: e00797.

37. Sheppard AE, Stoesser N, Wilson DJ, Sebra R, Kasarskis A, Anson LW, Giess A, Pankhurst $\sqcup$, Vaughan A, Grim CJ, et al. Nested Russian doll-like genetic mobility drives rapid dissemination of the Carbapenem resistance gene blaKPC. Antimicrob Agents Chemother. 2016;60:3767-78.

38. Willems S, Kampmeier S, Bletz S, Kossow A, Kock R, Kipp F, Mellmann A. Whole-genome sequencing elucidates epidemiology of nosocomial clusters of Acinetobacter baumannii. J Clin Microbiol. 2016;54:2391-4.

39. Halachev MR, Chan JZ, Constantinidou Cl, Cumley N, Bradley C, Smith-Banks M, Oppenheim B, Pallen MJ. Genomic epidemiology of a protracted hospital outbreak caused by multidrug-resistant Acinetobacter baumannii in Birmingham, England. Genome Med. 2014;6:70.

40. Lewis T, Loman NJ, Bingle L, Jumaa P, Weinstock GM, Mortiboy D, Pallen MJ. High-throughput whole-genome sequencing to dissect the epidemiology of Acinetobacter baumannii isolates from a hospital outbreak. J Hosp Infect. 2010;75:37-41.

Ready to submit your research? Choose BMC and benefit from:

- fast, convenient online submission

- thorough peer review by experienced researchers in your field

- rapid publication on acceptance

- support for research data, including large and complex data types

- gold Open Access which fosters wider collaboration and increased citations

- maximum visibility for your research: over $100 \mathrm{M}$ website views per year

At $\mathrm{BMC}$, research is always in progress.

Learn more biomedcentral.com/submission 